\title{
COMPARATIVE ANALYSIS OF NATURAL GAS ENGINE PARAMETERS WITH QUALITY AND QUANTITY CONTROL
}

\author{
Mikhail Shatrov $^{1}$, Aleksej Khatchiyan ${ }^{2}$, Vladimir Sinyavskiy ${ }^{3}$, \\ Ivan Shishlov ${ }^{4}$, Andrey Vakulenko ${ }^{5}$,
}

\begin{abstract}
Moscow Automobile and Road Construction State Technical University (MADI)
E-mail: ${ }^{1)}$ mikl-shatrov@yandex.ru, ${ }^{2}$ khachiyan28@bk.ru, ${ }^{3)}$ sinvlad@mail.ru,

4) astra510@yandex.ru, 5)ingener-avto@yandex.ru
\end{abstract}

Received 2014-06-30, accepted 2014-09-12

Parameters of natural gas engines were calculated with the aim to determine the optimal way of their working process organization. Analysis of calculations results demonstrated that quality power level control ensured the improvement of parameters of investigated engines. Calculations showed that compared with the diesel engine, the gas engine with quantity power level control, internal mixture formation and glow plug ignition of the gas-air mixture ensured the decrease of $\mathrm{CO}_{2}$ emissions by $26.8 \%$, and the natural gas engine with quality power level control, external mixture formation and gas-air mixture ignition by a small pilot portion of fine atomized diesel fuel supplied by a Common Rail fuel system - by $25.5 \%$. Therefore, one can choose one or another method of diesel engine conversion for operation on gas fuel considering available technical opportunities and with minimal expenses.

Diesel engine, gas engine, quality power control, quantity power control, dioxide carbon emissions calculation

\section{Introduction}

The paper is dedicated to comparative analysis of working processes of a diesel engine and four versions of natural gas engines to estimate their operation parameters and, first of all, carbon dioxide $\left(\mathrm{CO}_{2}\right)$ emissions which impacts of "greenhouse" effect. Four different ways of working processes of gas engines are analyzed in detail, in particular, quantity and quality methods of power level control and gas-air mixture inflammation.

\section{The goal of the research}

The goal was to choose the experimental data and computer modelling methods for the most suitable way of the organization of the working process of a natural gas powered engine which ensures the highest reduction of carbon dioxide emissions using 13-stage cycle according to the Rule ECE R49.

\section{Analysis of the working process organization in natural gas engines}

There may be many methods of the organization of the working process in natural gas powered engines. We shall analyze briefly few methods of conversion of diesel engines to operate on natural gas (Shatrov et al. 2013). These versions of gas engines will be later used for calculating their operation parameters. 
1. A natural gas engine designed on the base of a diesel engine having spark ignition and quantity power level control. This may be generally the stoichiometric gas engine or the lean mixture gas engine. Operation at the stoichiometric gas-air mixture results in considerable drop of fuel efficiency and hence - increase of $\mathrm{CO}_{2}$ emissions, thermal strain of the engine compared with the lean mixture gas engine. The lean mixture gas engine does not have these limitations and has one more advantage - its nitrogen oxides emissions level is much lower than that of the stoichiometric gas engine which makes it possible to avoid the use of the reduction catalyzer. Therefore, we used the lean mixture gas engine for our calculations.

2. A natural gas engine designed on the base of the diesel engine having spark plug or glow plug ignition and quality power level control. In this case, the problem of ignition of the gas-air mixture arises because, in contrast to diesel fuel, which has the self-ignition temperature about $350^{\circ} \mathrm{C}$, the self-ignition temperature of natural gas is about $700^{\circ} \mathrm{C}$. Therefore, to ignite the gas-air mixture, one has to use special heavy duty spark plugs or glow plugs and find experimentally the proper location place of the plug in relation to the gas injector sprays. Another approach is mounting the gas injectors in the intake system and using a pilot portion of diesel fuel for the inflammation of the gas-air mixture. One can use a traditional diesel fuel injection system and inject $15-25 \%$ of pilot diesel fuel portion. But the best solution based on the latest developments in diesel fuel supply systems is using the Common Rail system for injection of a pilot portion of diesel fuel. In this case, due to much higher injection pressure and computer control, it is possible to attain a fine atomization of the diesel fuel which makes it possible to decrease the portion of the diesel fuel to $3-5 \%$. Though to realize this method, one has to protect from overheating the injector nozzles because their cooling by fuel is poor due to small portions of fuel injected.

\section{Objects of investigation}

1. The base diesel engine (KAMAZ 74051-320) V-8 with piston diameter $\mathrm{D}=120$ $\mathrm{mm}$ and piston stroke $\mathrm{S}=120 \mathrm{~mm}$, compression ration $\varepsilon=17.0: 1$.

2. An engine designed on the base of the KAMAZ diesel engine, supplied with natural gas, having spark ignition and quantity power level control.

3. An engine designed on the base of the KAMAZ diesel engine, supplied with natural gas, with glow plug ignition and quality power level control.

4. A gas diesel engine designed on the base of the KAMAZ diesel engine with quality power level control and supply of $15 \mathrm{mg}$ of diesel fuel pilot portion injected by a traditional direct injection fuel supply system.

5. A gas diesel engine designed on the base of the KAMAZ diesel engine, with quality power level control and supply of $3 \mathrm{mg}$ of pilot portion of a fine atomized diesel fuel injected by a Common Rail fuel supply system with electro-hydraulic injectors. 


\section{Results of calculations of two natural gas powered engines with respect to load characteristics}

Parameters of natural gas engines with quality and quantity power level control were calculated by the model of joint operation of a gas engine with a turbocharger (Khatchiyan et al, 2010) at two engine operation modes: maximum power $(\mathrm{n}=2200 \mathrm{rpm})$ and maximum torque $(\mathrm{n}=1400 \mathrm{rpm})$.

Figure 1 shows the calculated characteristics of two natural gas engines having quality and quantity power level control versus mean effective pressure.
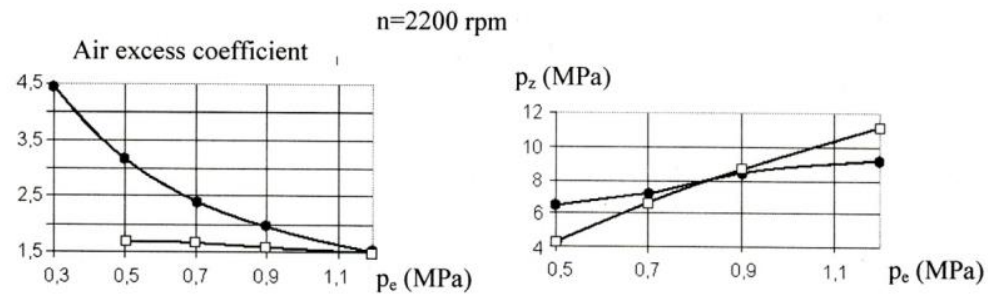

$\mathrm{P}_{\mathrm{k}}(\mathrm{MPa})$
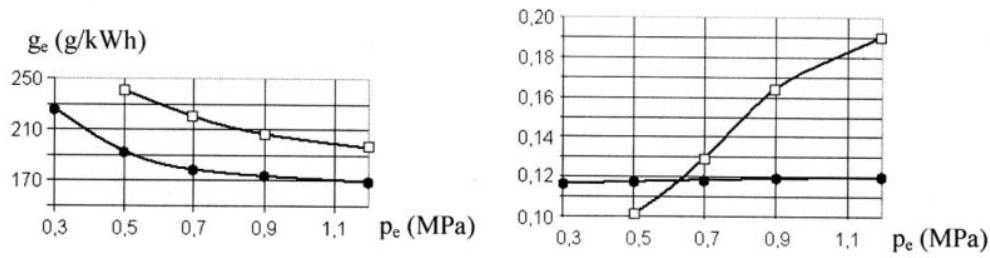

$\mathrm{n}=1400 \mathrm{rpm}$

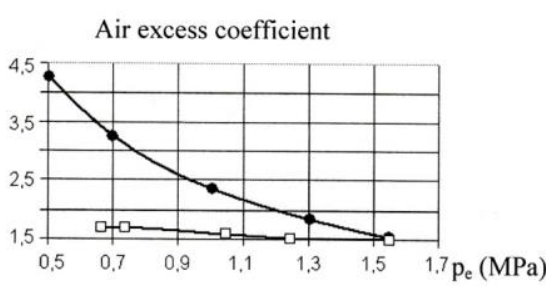

$\mathrm{p}_{z}(\mathrm{MPa})$
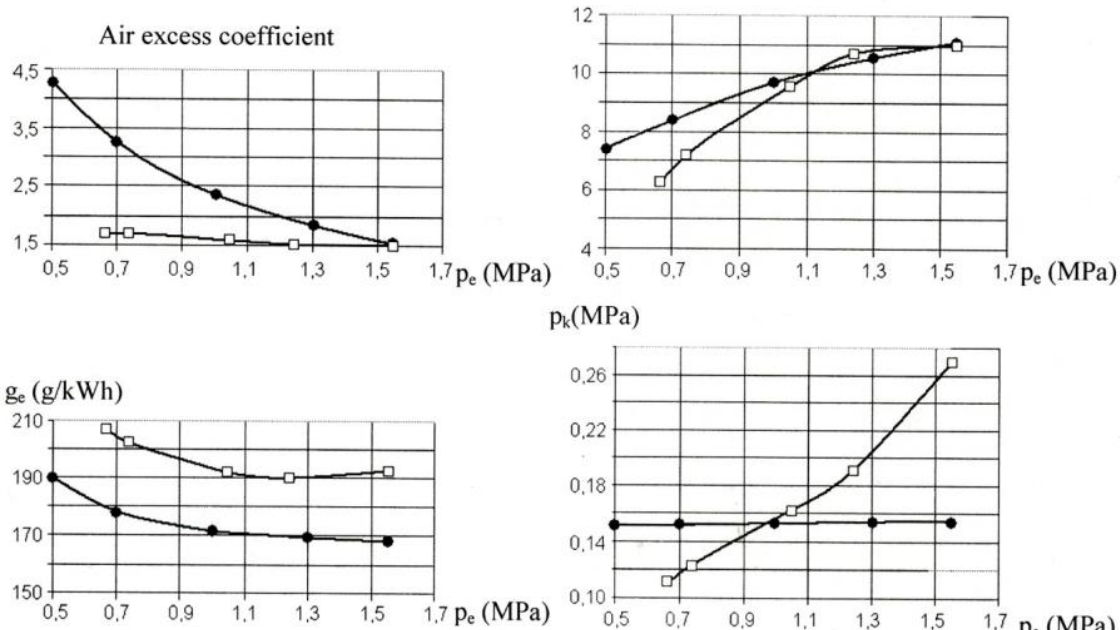

$\mathrm{p}_{\mathrm{k}}(\mathrm{MPa})$

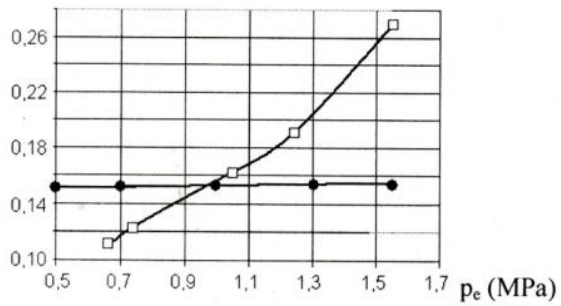

$\rightarrow$ Quality power level control
$\rightarrow-$ Quantity power level control

Fig. 1. Variation of calculated parameters of natural gas powered engines by load characteristics 
Analysis of the above mentioned characteristics demonstrates that in case of quality power level control of the gas engine, the lowest values of the brake specific fuel consumption $g_{e}$ are attained at both the engine speeds. The difference in $g_{e}$ is of course higher at low loads (higher air excess coefficient $\alpha$ ). The differences in other parameters are also linked with the nature of $\alpha$ variation.

One should note that equal values of the mean effective pressure were obtained in case of quantity power level control thanks to a higher boost pressure (a smaller turbine cross-section area was used).

Generally, it is possible to mark that quality power level control ensures the improvement of gas engines parameters.

For qualitative estimation of potential advantages of natural gas engines as regards their $\mathrm{CO}_{2}$ emissions, calculations of $\mathrm{CO}_{2}$ emissions were made for the above indicated KAMAZ diesel engine and four versions of natural gas engines designed on the base of the KAMAZ engine provided that these engines had equal fuel efficiency:

Condition of calculating $\mathrm{CO}_{2}$ emissions:

1. Comparison of $\mathrm{CO}_{2}$ emissions was made by the 13-stage cycle;

2. Low calorific value of diesel fuel $-42.56 \mathrm{MJ} / \mathrm{kg}$;

3. Carbon content in $1 \mathrm{~kg}$ of diesel fuel $-0.872 \mathrm{~kg}$;

4. Low calorific value of natural gas (taking into account the content of methane close to $100 \%$ ) $-50 \mathrm{MJ} / \mathrm{kg}$;

5. Carbon content in $1 \mathrm{~kg}$ of natural gas $-0.75 \mathrm{~kg}$;

6. When computing the dioxide carbon $\left(\mathrm{CO}_{2}\right)$ emissions in the gas engine with the diesel compression ratio, the difference in calorific value of both the fuels was taken into account;

7. The assumption was taken that the heat input for all operating modes was equal.

Natural gas consumption per hour in the gas diesel to obtain the same heat input as in the diesel engine was calculated by equation:

$$
G_{\text {gaseng }}=\left(G_{\text {diseng }}-G_{\text {pilot }} \times 30 \times n \times i \times 10^{-6}\right) \times\left(\frac{H_{U \text { gas }}}{H_{U \text { dis }}}\right),
$$

where $G_{\text {dis eng }}$ - diesel fuel consumption in the diesel engine $(\mathrm{kg} / \mathrm{h}), G_{\text {pilot }}-$ cycle fuel delivery of the diesel fuel pilot portion in the gas diesel engine (mg/cycle), $n-$ engine speed (rpm), $H_{U \text { dis- }}$ low calorific value of diesel fuel; $H_{U \text { gas }}$ low calorific value of natural gas; $i$ - number of cylinders (in our case, $i=8$ ).

Calculations of $\mathrm{CO}_{2}$ emissions were based on the content of carbon in the fuel. For the gas fuel, the content of carbon depends on its composition. The average results of six measurements carried out showed that the gas fuel available contained more than $98.5 \%$ of methane, other gases were: ethane, propane, butane, carbon dioxide and nitrogen.

When calculating $\mathrm{CO}_{2}$ emissions for the base diesel engine KAMAZ 74051320 , we used data obtained earlier during engine tests. 
The other engines were recalculated by their low calorific value using the data for diesel engine assuming that the engines considered had equal fuel efficiency.

\section{The results of $\mathrm{CO}_{2}$ calculations}

The results of $\mathrm{CO}_{2}$ calculations by the modes of the 13-stage engine cycle are shown in Tables 1, 2, 3, 4 (where $\mathrm{k}$ - weighting factor, accepted in compliance with the Rules R49 of the UN ECE).

The summary Table 5 indicates the values of specific emissions of dioxide carbon for the engines considered which shows the following:

- the decrease of $\mathrm{CO}_{2}$ emissions in the gas engine with quality power level control and glow plug ignition was $26.8 \%$ compared with the diesel engine;

- the decrease of $\mathrm{CO}_{2}$ emissions in the new generation gas engine was $25.5 \%$ compared with the diesel engine;

- the gas diesel engine with a Common Rail fuel injection system ensures the decrease of carbon dioxide emissions by additional 5.3\% compared with the gas diesel engine having a direct injection fuel supply system. Even higher effect of using natural gas is ensured when comparing with petrol engine (Khatchiyan et al., 2008).

Comparing the effects of decreasing emissions of the main greenhouse gas carbon dioxide using two methods of achieving the fuel efficiency equal to that of the base diesel engine: the gas engine with the gas-air mixture ignition by a glow plug and the gas engine with the gas-air mixture ignition by a small pilot portion of a fine atomized diesel fuel, one can say that both the approaches ensure a close value of reducing the emissions of $\mathrm{CO}_{2}$. Therefore, the choice between these two methods should be carried out taking into account other parameters:

1. Ensuring a stable operation with the fuel efficiency identical to that of a diesel engine in the whole range of performance modes of a vehicle.

2. The minimal development and production price and ensuring a reliable operation of the gas version of the base diesel

Based on the above stated and taking into consideration economic aspects, for carrying out the scientific-research work, we chose the working process of the natural gas engine with quality power level control and gas-air mixture ignition by a pilot portion of a fine atomized diesel fuel supplied by the Common Rail fuel system. It should be also mentioned that for automotive gas diesel engines, one should decrease heating of the injector nozzle, for example, by cooling it with circulating fuel. 
Table 1. 13-stage cycle of the base diesel engine

\begin{tabular}{|c|c|c|c|c|c|c|c|}
\hline n (rpm) & $\begin{array}{c}\mathrm{N}_{\mathrm{e}} \\
(\mathrm{kW})\end{array}$ & $\mathrm{k}$ & $\begin{array}{c}\mathrm{G}_{\text {fuel }} \\
(\mathrm{kg} / \mathrm{h})\end{array}$ & $\begin{array}{c}\mathrm{G}_{\text {air }} \\
(\mathrm{kg} / \mathrm{h})\end{array}$ & $\begin{array}{c}\mathrm{mCO}_{2}, \\
(\mathrm{~g} / \mathrm{h})\end{array}$ & $\begin{array}{c}\mathrm{mCO}_{2} * \mathrm{k} \\
(\mathrm{g} / \mathrm{h})\end{array}$ & $\begin{array}{l}\mathrm{N}_{\mathrm{e}}{ }^{* \mathrm{k}}, \\
(\mathrm{kW})\end{array}$ \\
\hline 600 & 0 & 0.083 & 1.10 & 215.10 & 3517 & 293 & 0 \\
\hline 1400 & 18.299 & 0.08 & 7.10 & 547.00 & 22701 & 1816 & 1.464 \\
\hline 1400 & 45.935 & 0.08 & 11.3 & 567.50 & 36130 & 2890 & 3.675 \\
\hline 1400 & 92.13 & 0.08 & 19.9 & 653.10 & 63627 & 5090 & 7.370 \\
\hline 1400 & 138.164 & 0.08 & 28.6 & 746.80 & 91444 & 7315 & 11.053 \\
\hline 1400 & 184.39 & 0.25 & 37.8 & 849.70 & 120859 & 30215 & 46.098 \\
\hline 600 & 0 & 0.0833 & 1.10 & 208.60 & 3517 & 293 & 0 \\
\hline 2200 & 215.663 & 0.1 & 53.7 & 1561.2 & 171697 & 17170 & 21.566 \\
\hline 2200 & 162.109 & 0.02 & 41.5 & 1450.6 & 132689 & 2654 & 3.242 \\
\hline 2200 & 107.802 & 0.02 & 30.4 & 1339.5 & 97199 & 1944 & 2.156 \\
\hline 2200 & 53.867 & 0.02 & 20.2 & 1122.0 & 64586 & 1292 & 1.077 \\
\hline 2200 & 21.713 & 0.02 & 14.2 & 1046.7 & 45402 & 908 & 0.434 \\
\hline 600 & 0 & 0.0833 & 1.10 & 208.60 & 3517 & 293 & 0 \\
\hline & & & & & & $\sum \mathrm{mCO}_{2} * \mathrm{k}$ & $\sum \mathrm{N}_{\mathrm{e}} * \mathrm{k}$ \\
\hline
\end{tabular}

Table 2. 13-stage cycle of the gas engine with quality power level control

\begin{tabular}{|c|c|c|c|c|c|c|c|}
\hline $\begin{array}{c}\mathrm{n} \\
(\mathrm{rpm})\end{array}$ & $\begin{array}{c}\mathrm{N}_{\mathrm{e}} \\
(\mathrm{kW})\end{array}$ & $\mathrm{k}$ & $\begin{array}{c}\mathrm{G}_{\text {fuel }} \\
(\mathrm{kg} / \mathrm{h})\end{array}$ & $\begin{array}{c}\mathrm{G}_{\mathrm{air}} \\
(\mathrm{kg} / \mathrm{h})\end{array}$ & $\begin{array}{c}\mathrm{mCO}_{2} \\
(\mathrm{~g} / \mathrm{h})\end{array}$ & $\begin{array}{c}\mathrm{mCO}_{2} * \mathrm{k} \\
(\mathrm{g} / \mathrm{h})\end{array}$ & $\begin{array}{l}\mathrm{N}_{\mathrm{e}} * \mathrm{k}, \\
(\mathrm{kW})\end{array}$ \\
\hline 600 & 0 & 0.0833 & 0.936 & 215.1 & 2575 & 214 & 0 \\
\hline 1400 & 18.299 & 0.08 & 6.044 & 547.0 & 16620 & 1330 & 1.464 \\
\hline 1400 & 45.935 & 0.08 & 9.619 & 567.5 & 26451 & 2116 & 3.675 \\
\hline 1400 & 92.130 & 0.08 & 16.939 & 653.1 & 46582 & 3727 & 7.370 \\
\hline 1400 & 138.164 & 0.08 & 24.344 & 746.8 & 66947 & 5356 & 11.053 \\
\hline 1400 & 184.390 & 0.25 & 32.175 & 849.7 & 88482 & 22121 & 46.098 \\
\hline 600 & 0 & 0.0833 & 0.936 & 208.6 & 2575 & 214 & 0 \\
\hline 2200 & 215.663 & 0.1 & 45.709 & 1561.2 & 125701 & 12570 & 21.566 \\
\hline 2200 & 162.109 & 0.02 & 35.325 & 1450.6 & 97143 & 1943 & 3.242 \\
\hline 2200 & 107.802 & 0.02 & 25.876 & 1339.5 & 71160 & 1423 & 2.156 \\
\hline 2200 & 53.867 & 0.02 & 17.194 & 1122.0 & 47284 & 946 & 1.077 \\
\hline 2200 & 21.713 & 0.02 & 12.087 & 1046.7 & 33239 & 665 & 0.434 \\
\hline 600 & 0 & 0.0833 & 0.936 & 208.6 & 2575 & 214 & 0 \\
\hline & & & & & & $\sum \mathrm{mCO}_{2} * \mathrm{k}$ & $\sum \mathrm{N}_{\mathrm{e}} * \mathrm{k}$ \\
\hline
\end{tabular}




\begin{tabular}{|c|c|c|c|c|c|c|c|c|c|c|c|c|c|c|c|}
\hline $\begin{array}{l}* \\
\stackrel{*}{*} \frac{3}{y}\end{array}$ & 0 & $\begin{array}{l}\text { to } \\
\stackrel{+}{+} . \\
\text {. }\end{array}$ & $\mid \begin{array}{l}n \\
\sigma \\
\dot{\sigma} \\
\dot{n}\end{array}$ & 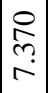 & $\mid \begin{array}{l}n \\
\tilde{n} \\
= \\
=\end{array}$ & $\begin{array}{l}\infty \\
8 \\
0 \\
\dot{b} \\
\dot{0}\end{array}$ & 0 & 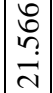 & 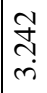 & 党 & 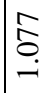 & 守 & 0 & 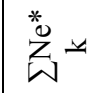 & 吕 \\
\hline 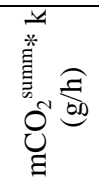 & 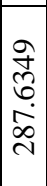 & 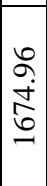 & 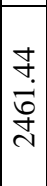 & 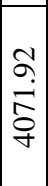 & 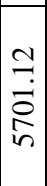 & $\mid \begin{array}{l}\stackrel{2}{a} \\
\stackrel{a}{a} \\
\overrightarrow{2}\end{array}$ & 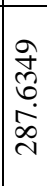 & 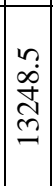 & 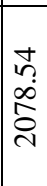 & $\begin{array}{l}\infty \\
\infty \\
\infty \\
\infty \\
n \\
n\end{array}$ & $\begin{array}{l}0 \\
? \\
\infty \\
\infty \\
0\end{array}$ & 宔 & 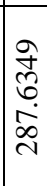 & W & $\begin{array}{l}\tilde{\infty} \\
\infty \\
\tilde{n} \\
\tilde{\sigma} \\
\tilde{n}\end{array}$ \\
\hline 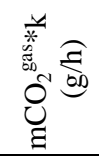 & 0 & $\begin{array}{l}2 \\
2 \\
\infty \\
\infty \\
n\end{array}$ & 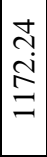 & 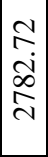 & 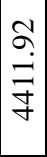 & 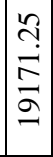 & 0 & 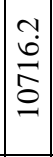 & $\begin{array}{c}\infty \\
0 \\
i \\
i \\
i n \\
-1\end{array}$ & 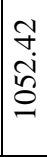 & ì & ปे & 0 & W & $=\frac{m}{\frac{m}{\sim}}$ \\
\hline 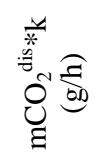 & 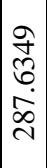 & 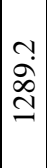 & 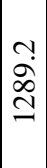 & 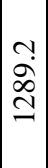 & 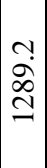 & 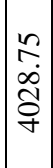 & 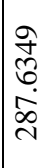 & $\begin{array}{l}n \\
\tilde{n} \\
\tilde{n} \\
\end{array}$ & $\begin{array}{l}0 \\
+ \\
0 \\
0 \\
0 \\
i n\end{array}$ & $\begin{array}{l}0 \\
+ \\
0 \\
0 \\
0 \\
i n\end{array}$ & \begin{tabular}{l}
0 \\
+ \\
\multirow{2}{0}{} \\
0 \\
0
\end{tabular} & $\begin{array}{l}0 \\
+ \\
8 \\
8 \\
\text { in }\end{array}$ & 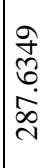 & 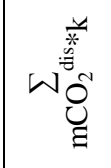 & 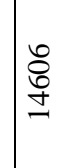 \\
\hline 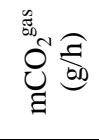 & 0 & $\begin{array}{l}\text { ते } \\
\infty \\
+\end{array}$ & 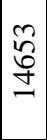 & 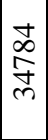 & $\begin{array}{l}\frac{a}{+} \\
i n \\
n\end{array}$ & $\begin{array}{l}2 \\
0 \\
0 \\
0 \\
1\end{array}$ & 0 & 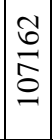 & $\begin{array}{l}8 \\
0 \\
0 \\
\infty \\
1\end{array}$ & $\begin{array}{l}\overrightarrow{\widetilde{V}} \\
\mathbb{N} \\
\text { ñ }\end{array}$ & 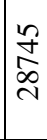 & 荨 & 0 & & \\
\hline 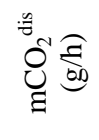 & $\stackrel{n}{n}$ & $\frac{n}{6}$ & $\underline{n}$ & 旾 & 旾 & $\underline{n}$ & 吕 & $\mid \begin{array}{l}0 \\
\tilde{2} \\
\tilde{2}\end{array}$ & $\begin{array}{l}2 \\
\hat{n} \\
\tilde{n} \\
\end{array}$ & $\begin{array}{l}2 \\
\tilde{n} \\
\tilde{n} \\
\sim\end{array}$ & 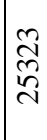 & 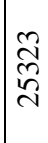 & $\frac{n}{n}$ & & \\
\hline ن & $\frac{\vec{n}}{\vec{n}}$ & 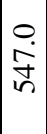 & 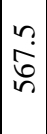 & $\begin{array}{l}- \\
\tilde{n} \\
\tilde{6}\end{array}$ & 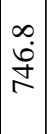 & $\begin{array}{l}2 \\
\dot{+} \\
\infty\end{array}$ & 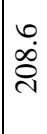 & 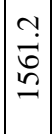 & 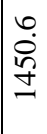 & $\begin{array}{l}n \\
\tilde{m} \\
m\end{array}$ & $\begin{array}{l}0 \\
\text { İ } \\
\text { ב }\end{array}$ & $\begin{array}{l}0 \\
0 \\
0 \\
0\end{array}$ & $\begin{array}{l}0 \\
0 \\
0 \\
0\end{array}$ & & \\
\hline 怠 & 0 & 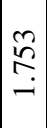 & 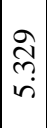 & 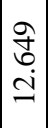 & 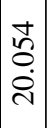 & 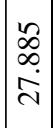 & 0 & $\left|\begin{array}{l}\infty \\
0 \\
0 \\
\infty \\
\infty \\
m\end{array}\right|$ & $\begin{array}{l}0 \\
\infty \\
n \\
\infty \\
\sim\end{array}$ & $\begin{array}{l}n \\
2 \\
a \\
2\end{array}$ & 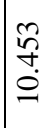 & 年 & 0 & & \\
\hline ن & $\begin{array}{l}\infty \\
\stackrel{0}{8} \\
0\end{array}$ & $\begin{array}{l}0 \\
0 \\
0 \\
8 \\
0 \\
0\end{array}$ & $\begin{array}{l}0 \\
0 \\
8 \\
0 \\
0 \\
0\end{array}$ & $\mid \begin{array}{l}0 \\
⿱ 丷 \\
8 \\
0 \\
0 \\
0\end{array}$ & $\begin{array}{l}0 \\
⿱ \\
\delta \\
0 \\
0 \\
0\end{array}$ & $\begin{array}{l}0 \\
0 \\
8 \\
0 \\
0 \\
0\end{array}$ & $\stackrel{\infty}{0}$ & $\mid$\begin{tabular}{l}
0 \\
\hdashline \\
\hdashline \\
0 \\
0
\end{tabular} & $\begin{array}{l}0 \\
0 \\
8 \\
0 \\
0\end{array}$ & $\begin{array}{l}0 \\
0 \\
8 \\
0 \\
0 \\
0\end{array}$ & 苑 & 苑 & $\frac{\infty}{8}$ & & \\
\hline$\not$ & $\begin{array}{l}\stackrel{M}{0} \\
\infty \\
0 \\
0\end{array}$ & $\begin{array}{l}\infty \\
\stackrel{0}{0} \\
0\end{array}$ & $\begin{array}{l}\infty \\
\stackrel{\infty}{0} \\
\dot{0}\end{array}$ & $\begin{array}{l}\infty \\
0 \\
0 \\
0\end{array}$ & \begin{tabular}{|l|}
$\infty$ \\
0 \\
0 \\
0
\end{tabular} & 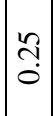 & $\begin{array}{l}0 \\
\tilde{\infty} \\
0 \\
0 \\
0\end{array}$ & $\overrightarrow{0}$ & $\begin{array}{l}\Omega \\
0 \\
0\end{array}$ & $\begin{array}{l}\Omega \\
0 \\
0\end{array}$ & $\begin{array}{l}\sigma \\
0 \\
0\end{array}$ & $\begin{array}{l}\sigma \\
0 \\
0\end{array}$ & $\begin{array}{l}m \\
\stackrel{\infty}{\infty} \\
\stackrel{0}{0}\end{array}$ & & \\
\hline$z^{0} \bar{z}$ & 0 & $\begin{array}{l}\stackrel{2}{\grave{\lambda}} \\
\infty \\
-\end{array}$ & $\begin{array}{l}n \\
\sigma \\
\tilde{\gamma} \\
\gamma\end{array}$ & 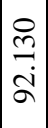 & 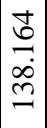 & 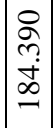 & 0 & 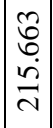 & $\begin{array}{l}8 \\
0 \\
\text { i } \\
0\end{array}$ & $\begin{array}{c}1 \\
\delta \\
\infty \\
5 \\
0 \\
0\end{array}$ & $\begin{array}{l}\hat{\delta} \\
\infty \\
\dot{n}\end{array}$ & $\frac{m}{i}$ & 0 & & \\
\hline$=\widehat{\widehat{\Xi}}$ & 8 & ষ & \& & 8 & 8 & 焉 & 8 & $\begin{array}{l}\text { ¿̊ } \\
\text { ন }\end{array}$ & તิ & ণิ & స్ & ๙ & 8 & & \\
\hline
\end{tabular}




\begin{tabular}{|c|c|c|c|c|c|c|c|c|c|c|c|c|c|c|c|}
\hline 蒙妾 & 0 & 苂 & $\mid \begin{array}{c}n \\
6 \\
\dot{r} \\
\end{array}$ & ? & $\begin{array}{l}n \\
0 \\
=\end{array}$ & $\begin{array}{l}\infty \\
\stackrel{0}{0} \\
\dot{0} \\
+\end{array}$ & 0 & 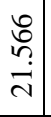 & $\underset{\stackrel{Y}{4}}{\stackrel{n}{m}}$ & 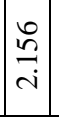 & 合 & 志 & 0 & $\begin{array}{l}\frac{y}{*} \\
\tilde{0} \\
\text { 出 }\end{array}$ & $\frac{\infty}{\infty}$ \\
\hline 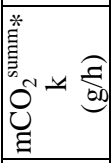 & & $\stackrel{2}{2}$ & $\mid \begin{array}{l}\infty \\
\infty \\
\sim\end{array}$ & $\underset{\substack{2\\
}}{\stackrel{0}{2}}$ & $\stackrel{2}{y}$ & $\begin{array}{l}\hat{n} \\
\text { ते }\end{array}$ & $\stackrel{N}{\approx}$ & 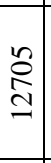 & 尽 & 号 & $\stackrel{n}{a}$ & â & $\stackrel{\sim}{\sim}$ & 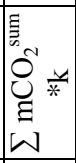 & 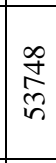 \\
\hline 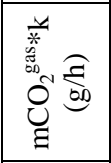 & $\stackrel{\Delta}{\sim}$ & 寻 & $\widehat{\widehat{\Omega}}$ & $\begin{array}{l}\infty \\
\infty \\
n \\
m\end{array}$ & $\frac{\hat{\sigma}}{n}$ & $\vec{n}$ & $\vec{\nabla}$ & $\begin{array}{l}\stackrel{\partial}{\Delta} \\
\text { I }\end{array}$ & $\begin{array}{l}8 \\
\infty \\
-\end{array}$ & 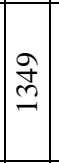 & $\underset{\infty}{\mathbb{N}}$ & $\bar{n}$ & $\stackrel{ \pm}{\sim}$ & 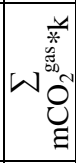 & 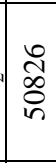 \\
\hline 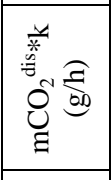 & $\stackrel{\infty}{n}$ & $\begin{array}{l}\infty \\
\sim \\
\sim\end{array}$ & $\begin{array}{l}\infty \\
\\
\end{array}$ & $\begin{array}{l}\infty \\
\infty \\
\sim\end{array}$ & 文 & $\underset{\infty}{\infty}$ & $\infty$ & ஜ & $\overline{0}$ & $\overline{0}$ & $\overline{0}$ & $\overline{0}$ & $\stackrel{\infty}{\curvearrowleft}$ & 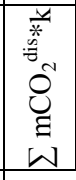 & নু \\
\hline 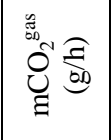 & $\frac{\pi}{n}$ & \begin{tabular}{l}
8 \\
$\stackrel{8}{1}$ \\
\multirow{2}{J}{} \\
\end{tabular} & 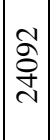 & 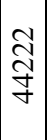 & $\begin{array}{l}\infty \\
\infty \\
\mathfrak{f} \\
b\end{array}$ & $\frac{2}{\mathfrak{I}}$ & 志 & 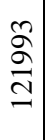 & $\begin{array}{c}n \\
\tilde{f} \\
\tilde{\sigma} \\
\end{array}$ & $\begin{array}{l}\frac{N}{5} \\
\frac{5}{5}\end{array}$ & $\left|\begin{array}{c}0 \\
0 \\
\tilde{f} \\
\dot{+}\end{array}\right|$ & $\mid \begin{array}{l}n \\
\tilde{n} \\
2 \\
\end{array}$ & 志 & & \\
\hline 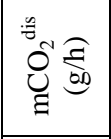 & $\overline{8}$ & $\begin{array}{l}\tilde{z} \\
\text { ñ }\end{array}$ & 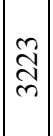 & 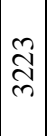 & ָิ & ลิ & $\overline{\mathbf{b}}$ & $\mid \begin{array}{l}n \\
0 \\
0 \\
n\end{array}$ & 䘾 & $\begin{array}{l}n \\
0 \\
0 \\
n\end{array}$ & $\begin{array}{l}n \\
0 \\
0 \\
n\end{array}$ & $\mid \begin{array}{l}n \\
8 \\
0 \\
n\end{array}$ & $\overline{\hat{\sigma}}$ & & \\
\hline ن & $\frac{\vec{n}}{\sqrt{n}}$ & \begin{tabular}{c}
0 \\
\multirow{+}{*}{} \\
$\dot{n}$
\end{tabular} & $\begin{array}{l}n \\
6 \\
n\end{array}$ & 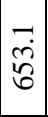 & $\begin{array}{l}\infty \\
\dot{0} \\
\stackrel{+}{1}\end{array}$ & $\begin{array}{l}\hat{a} \\
\dot{\infty}\end{array}$ & 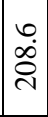 & 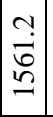 & $\begin{array}{l}0 \\
\dot{0} \\
\dot{8} \\
=\end{array}$ & $\begin{array}{l}n \\
\dot{m} \\
m \\
2\end{array}$ & $\begin{array}{l}0 \\
\dot{I} \\
\text { I }\end{array}$ & $\begin{array}{l}r \\
\dot{0} \\
0 \\
0\end{array}$ & 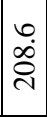 & & \\
\hline 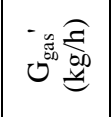 & 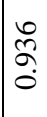 & $\begin{array}{c}\infty \\
\infty \\
i n \\
i n\end{array}$ & $\left|\begin{array}{c|}\overline{0} \\
\infty \\
\infty\end{array}\right|$ & $\mid \begin{array}{l}\infty \\
0 \\
0 \\
0 \\
-\end{array}$ & \begin{tabular}{l}
$\infty$ \\
$\infty$ \\
+ \\
\multirow{2}{d}{}
\end{tabular} & $\frac{\bar{n}}{m}$ & 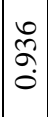 & $\mid \begin{array}{l}\vec{b} \\
\tilde{f} \\
\dot{f}\end{array}$ & $\begin{array}{l}0 \\
\vdots \\
\hat{m} \\
m\end{array}$ & $\left|\begin{array}{l}\infty \\
\sim \\
\sim \\
\sim \\
\sim\end{array}\right|$ & $\mid \begin{array}{l}0 \\
0 \\
0 \\
i n \\
-1\end{array}$ & $\mid \begin{array}{l}2 \\
\tilde{2} \\
\dot{0}\end{array}$ & 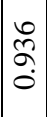 & & \\
\hline 氖 & $\begin{array}{l}0 \\
\text { గొ } \\
8 \\
8 \\
0\end{array}$ & 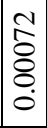 & 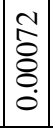 & 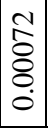 & 䇂 & 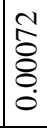 & $\mid \begin{array}{l}0 \\
⿱ 0 \\
8 \\
8 \\
0 \\
0\end{array}$ & $\mid \begin{array}{c}1 \\
\hat{\delta} \\
0 \\
0 \\
0\end{array}$ & $\mid \begin{array}{c}1 \\
\hat{8} \\
8 \\
0 \\
0\end{array}$ & 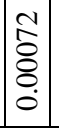 & 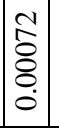 & 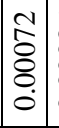 & $\begin{array}{l}0 \\
\text { O̊ } \\
8 \\
8 \\
0\end{array}$ & & \\
\hline 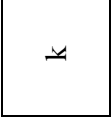 & $\begin{array}{l}m \\
\infty \\
\infty \\
0 \\
0\end{array}$ & $\stackrel{\infty}{\circ}$ & 足 & $\begin{array}{l}\infty \\
0 \\
0 \\
0\end{array}$ & $\begin{array}{l}\infty \\
0 \\
0\end{array}$ & $\stackrel{2}{n}$ & $\mid \begin{array}{l}0 \\
\tilde{O} \\
0 \\
0 \\
0\end{array}$ & $\overline{0}$ & $\begin{array}{l}1 \\
0 \\
0\end{array}$ & $\begin{array}{l}\sigma \\
0 \\
0\end{array}$ & $\mid \begin{array}{l}\sigma \\
0 \\
0\end{array}$ & $\begin{array}{l}\sigma \\
0 \\
0\end{array}$ & $\begin{array}{l}m \\
\hat{\infty} \\
0 \\
0 \\
0\end{array}$ & & \\
\hline$z^{0} \frac{z}{y}$ & 0 & 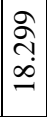 & $\mid \begin{array}{l}n \\
\tilde{\alpha} \\
i \\
\gamma\end{array}$ & $\begin{array}{c}\stackrel{c}{m} \\
\stackrel{a}{a}\end{array}$ & $\begin{array}{l}t \\
0 \\
0 \\
0 \\
-\end{array}$ & 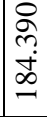 & 0 & $\begin{array}{l}c \\
\dot{b} \\
\dot{n} \\
\end{array}$ & $\mid \begin{array}{c}2 \\
0 \\
i \\
0 \\
-\end{array}$ & $\left|\begin{array}{l}\mathcal{O} \\
0 \\
\infty \\
\vdots \\
0 \\
-\end{array}\right|$ & $\begin{array}{l}\hat{\delta} \\
\infty \\
\dot{n} \\
\hat{n}\end{array}$ & $\frac{\pi}{i}$ & 0 & & \\
\hline$\underset{\Xi}{\stackrel{\Xi}{\Xi}}$ & 8 & 字 & \& & \& & \& & \& & 8 & ¿̊. & 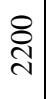 & ¿̊. & ¿ి & 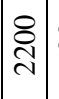 & 8 & & \\
\hline
\end{tabular}


Table 5. Summary Table

\begin{tabular}{|c|l|c|}
\hline \multicolumn{1}{|c|}{ Engine type } & \multicolumn{1}{|c|}{$\begin{array}{c}\text { Specific } \mathrm{CO}_{2} \\
\text { emissions }(\mathrm{g} / \mathrm{kWh})\end{array}$} \\
\hline 1 & \multicolumn{1}{|c|}{ Diesel engine } & 734.44 \\
\hline 2 & $\begin{array}{l}\text { Natural aspirated gas engine with spark ignition and quantity } \\
\text { power level control }\end{array}$ & 624.00 \\
\hline 3 & $\begin{array}{l}\text { Gas engine with glow plug ignition and quality power level } \\
\text { control }\end{array}$ & 538.30 \\
\hline 4 & $\begin{array}{l}\text { Gas engine with quality power level control and supply of a pilot } \\
\text { portion of diesel fuel 15 mg/cycle (direct injection fuel supply } \\
\text { system) }\end{array}$ & 578.176 \\
\hline 5 & $\begin{array}{l}\text { Gas engine with quality power level control and supply of a pilot } \\
\text { portion of a fine atomized diesel fuel 3 mg/cycle (Common Rail } \\
\text { fuel supply system) }\end{array}$ & 547.683 \\
\hline
\end{tabular}

\section{Conclusions}

Comparison of operation parameters of the diesel engine with four versions of natural gas supplied engines demonstrated, that the gas engine with the glow plug ignition and quality power level control, and the gas engine with the quality power level control and supply of a pilot portion of a fine atomized diesel fuel by the Common Rail system, ensure the lowest level of $\mathrm{CO}_{2}$ emissions (approximately by $26 \%$ ) compared to the diesel engine on condition of equal amount of heat inputted into the cylinders.

\section{References}

1. Khatchijan A.S., Sinyavskiy V.V., Shishlov I.G., Karpov D.M. 2010. Modelirovanie pokazatelej i harakteristik dvigatelej, pitaemyh prirodnym gazom. Transport na al'ternativnom toplive, 3(15), 14-19. (Khatchijan A.S., Sinyavskiy V.V., Shishlov I.G., Karpov D.M. 2010. Modeling of Parameters and Characteristics of Natural Gas Powered Engines. Transport Running on Alternative Fuel, 3(15), 14-19)

2. Khatchijan A.S., Shishlov I.G., Vakulenko A.V. 2008. Avtomobil'nyj transport i parnikovyj effect. Transport na al'ternativnom toplive, 2 (2), 68-70 (Khatchijan A.S., Shishlov I.G., Vakulenko A.V. 2008. Automobile Transport and Greenhouse Effect. Transport Running on Alternative Fuel, 2(2), 68-70)

3. Shatrov M.G., Khatchijan A.S., Shishlov I.G., Vakulenko A.V. 2013. Analiz sposobov konvertacii avtomobil'nyh dizeli na pitanie prirodnym gazom. Transport na al'ternativnom toplive, 4 (34), 29-32 (Khatchijan A.S., Shishlov I.G., Vakulenko A.V. 2008. Analysis of Conversion Methods of Automotive Diesel Engines to be Powered with Natural Gas. Transport Running on Alternative Fuel, 4(34), 29-32)

4. Emission Test Cycles: ECE R49 - DieselNet [cited 20 May 2014]. Available at: http://www.dieselnet.com/standards/cycles/ece_r49.php 